\title{
THE POSSIBLE IMPACT OF L5 MAGNETOGRAMS ON NON-POTENTIAL SOLAR CORONAL MAGNETIC
} FIELD SIMULATIONS

\author{
Marion Weinzierl ${ }^{1}$, Duncan H. Mackay ${ }^{2}$, Anthony R. Yeates ${ }^{1}$, and Alexei A. Pevtsov ${ }^{3}$ \\ ${ }^{1}$ Department of Mathematical Sciences, Durham University South Road, Durham DH1 3LE, UK; marion.weinzierl@durham.ac.uk \\ ${ }^{2}$ School of Mathematics and Statistics, University of St. Andrews North Haugh, St. Andrews, Fife KY16 9SS, UK \\ ${ }^{3}$ National Solar Observatory 3010 Coronal Loop, sunspot NM 88349, USA \\ Received 2016 May 12; revised 2016 July 8; accepted 2016 July 9; published 2016 September 8
}

\begin{abstract}
The proposed Carrington-L5 mission would bring instruments to the L5 Lagrange point to provide us with crucial data for space weather prediction. To assess the importance of including a magnetograph, we consider the possible differences in non-potential solar coronal magnetic field simulations when magnetograph observations are available from the L5 point, compared with an L1-based field of view (FOV). A timeseries of synoptic radial magnetic field maps is constructed to capture the emergence of two active regions from the L5 FOV. These regions are initially absent in the L1 magnetic field maps, but are included once they rotate into the L1 FOV. Non-potential simulations for these two sets of input data are compared in detail. Within the bipolar active regions themselves, differences in the magnetic field structure can exist between the two simulations once the active regions are included in both. These differences tend to reduce within 5 days of the active region being included in L1. The delayed emergence in L1 can, however, lead to significant persistent differences in long-range connectivity between the active regions and the surrounding fields, and also in the global magnetic energy. In particular, the open magnetic flux and the location of open magnetic footpoints, are sensitive to capturing the real-time of emergence. These results suggest that a magnetograph at L5 could significantly improve predictions of the nonpotential corona, the interplanetary magnetic field, and of solar wind source regions on the Sun.
\end{abstract}

Key words: magnetohydrodynamics (MHD) - Sun: corona - Sun: coronal mass ejections (CMEs) - Sun: magnetic fields

\section{INTRODUCTION}

Space weather describes the many effects that solar variability can have on the Earth and the near-Earth environment. While it has long been known that eruptive solar phenomena such as solar flares, Coronal Mass Ejections, or filament eruptions can lead to severe space weather events on Earth (e.g., the shorting of power grids, disruption in communications and GPS systems, or even the loss of satellites), it is only in recent years that its international importance has been recognized (see the report by Schrijver et al. 2015). This has led to the proposed Carrington-L5 mission to place a satellite at the L5 Lagrange point, $60^{\circ}$ behind the orbit of the Earth (Trichas et al. 2015). Such a satellite could provide an additional early warning of 5-6 days of the active regions on the Sun as they rotate toward the Earth. The payload for such a mission is still under discussion, but proposed instruments include both remote-sensing instruments (e.g., coronagraph, heliospheric imager, Extreme Ultraviolet Imager, and line-of-sight magnetograph) and in situ capabilities (for measuring particles and fields). This paper continues the study started by Mackay et al. (2016) to consider the importance of a normal component magnetograph for understanding the nature of the Sun's global non-potential field. For a discussion of the wide range of global non-potential models currently in use, see Mackay \& Yeates (2012, Section 3).

Mackay et al. (2016) presented a theoretical study to consider what improvement could be obtained in global nonpotential modeling of the Sun if magnetograph data were available from the L5 Lagrange point in addition to those obtained from Earth. Their study used synthetic bipole data sets that first described a "reference Sun" simulation where random magnetic bipoles were allowed to emerge across the entire solar surface at any given time. From this, two "limited data" simulations were then constructed where bipoles were only included when they could be seen from (i) an Earth-based magnetograph and (ii) both Earth- and L5-based magnetographs. In each case, the resulting non-potential solar corona was modelled for two solar cycles (22 years), and global quantities such as surface flux, total magnetic energy, volume electric current, open flux, and the number of flux ropes were studied. Their results showed that if magnetograph data were available from the L5 point in conjunction with the Earth, this would significantly increase the accuracy of the global quantities mentioned above in non-potential modeling by anywhere from $26 \%$ to $40 \%$ compared with the existing situation which relies on only Earth-based data.

The study described above considered what increase in accuracy can occur over long time periods if magnetograph observations are available from the L5 point. This was carried out with a purely theoretical approach, using synthetic bipole data sets and considering only global quantities. For the present paper, we will consider a much more in-depth analysis using an observationally derived data set. We will consider in detail what differences occur in the global and local magnetic field structure when active regions emerge at their correct time from the L5 field of view (FOV), but rotate into the L1 FOV at a later time. Thus, in the present paper we carry out a shorter time but a more local analysis.

The structure of the paper is as follows. In Section 2, the construction of the observationally derived data representing both the L1 and L5 viewpoints is described. In Section 3, the technique for simulating the non-potential global coronal field is described. Following this, the global (Section 4) and then local (Section 5) properties of the coronal fields in both the L1 
and L5 simulations are compared and their differences identified. Finally, the conclusions are given in Section 6.

\section{DATA SETS}

To consider in detail how observations of active region emergence that are captured from the L5 FOV would increase the accuracy of global modeling, two synthetic data sets were constructed from SOLIS/VSM near-real-time (daily) synoptic maps. These synoptic maps are updated daily, using the present Carrington rotation (CR) along with the previous rotation to produce a best estimate of the global magnetic field in the solar photosphere. Within these maps, only longitudes visible from Earth are updated, with a $\cos ^{4} \phi$ dependence weighted to the current central meridian. Non-visible parts are taken from previous observations under the assumption that the field has not evolved in the intervening time. More advanced methods of constructing a timeseries of the global magnetic field do exist, where the longitudes that are not visible are evolved using surface flux transport models (for example, see Arge et al. 2010; Henney et al. 2012; Arge et al. 2013; Upton \& Hathaway 2014). However, for the present paper, this extra complexity is not required. Our synthetic data sets cover three successive Carrington rotations, CR 2146-2148.

To create the artificial data sets, the synoptic data are first translated to represent what would be seen from a magnetograph at the L5 point. Next, the data are translated back to the L1 point. However, to represent the later observation from this longitude, we removed two active regions that emerged within the L5 FOV, but which should not yet be visible from L1. In the L1 data set, these are replaced by random noise with amplitude and spatial properties similar to areas of quiet Sun on the same map. Once they rotate into the L1 FOV, they are then included in the L1 data set with the properties that they have at this later time. In a crude manner, this simulates the effect of active regions being observed later from L1 than from L5. The new data are assimilated gradually over one day so that there is no sudden change in the electric and magnetic fields. As in Weinzierl et al. (2016), this is done by linearly interpolating the electric fields, which are computed from the daily magnetograms as described in Section 3. The two active regions selected for this test are compact and wellseparated from the surrounding magnetic fields. Their removal does not affect the magnetic flux imbalance over the corresponding synoptic maps.

Figure 1 illustrates the L1 (right column) and L5 (left column) data sets, which extend over a 48-day period. In each case, the rotation of the Sun was removed and the data sets were aligned for ease of comparison. On day 0 , both data sets are assumed to be identical to one another and they follow an identical evolution until day 15 . For the non-potential simulations carried out in Sections 4 and 5, this is used as a ramp-up phase to build up electric currents in the corona. On day 15 , an active region starts emerging in the northern hemisphere and is fully emerged by day 17 (denoted by the red circle), but it is only visible from the L5 FOV. The presence of the active region in L5 and the corresponding absence in L1 can be clearly seen. It takes a further 4 days of solar rotation (red arrow) until the active region becomes fully visible from L1 on day 21 (red circle, right column), at which time its properties now match those in L5. While the properties match at this time, the L5 simulation will have included four additional days of evolution of the active region, during which stress and energy will have been built up in the coronal magnetic field. From day 21, the radial field at the photosphere in both cases follows an identical evolution until day 27, when a second active region emerges within the L5 FOV (fully emerged by day 29). Once again, it is absent in L1 at this time and is not fully visible there until day 35 . Thus, once again, a number of days of evolution of the active region and of injection of magnetic energy and helicity into the corona are missed. From day 35 onwards, both the L5 and L1 simulations follow an identical evolution.

It should be noted at this point that the manner in which the data sets are constructed results in minimal differences between L1 and L5. They are assumed different only at the location of the two active regions, and are identical elsewhere. In reality, the magnetic field would be continually evolving at all locations at all times. However, by constructing the data set in this manner, we can more clearly quantify what effect the missing active region evolution has on the corona by isolating these effects relative to the surrounding fields. Therefore, we are considering the least possible differences. Because the L5 data set more accurately captures the evolution of the active regions compared with L1, for the purposes of the present study we will regard it as being the "real" Sun, while L1 is our current best approximation. In the next section, the technique used to simulate both the global non-potential photospheric and coronal magnetic fields is discussed.

\section{NON-POTENTIAL SIMULATION TECHNIQUE}

Figure 2 depicts the simulated three-dimensional (3D) nonpotential magnetic field of the two active regions on days where they are completely visible in both the L1 and L5 FOV. From a visual inspection of both the left (day 21) and right (day 36) columns, it can be seen that the photospheric field is identical in both cases. In contrast, there are obvious differences in the connectivity and structure of the coronal magnetic field between the L1 (top row) and L5 (bottom row) simulations. Although this image shows the local structure of the active regions, there are also global differences in the coronal magnetic field which are indicated exemplarily by the global magnetic energy that is given for each case, as the connectivity of the active regions to the surrounding field is also different. Therefore, even though the only difference between the two simulations is the local time of emergence of the active regions, it can lead to not only local but also to global differences, as we will see in Section 4. We first describe our simulation technique used to produce the non-potential fields.

\subsection{Coronal Model}

We simulate the solar coronal evolution using the magnetofrictional (MF) technique to follow the evolution of the largescale magnetic field. The method was developed by van Ballegooijen et al. (2000) and was applied to the global corona by Yeates et al. (2008). Instead of solving the full MHD momentum equation, the velocity $\boldsymbol{v}$ is approximated by the magneto-frictional form introduced by Yang et al. (1986), namely,

$$
\boldsymbol{v}=\nu^{-1}\left(\boldsymbol{J} \times \boldsymbol{B} / B^{2}\right)
$$

where $\boldsymbol{J}=\nabla \times \boldsymbol{B}$ and $\nu$ is a friction coefficient. The 3D magnetic field $\boldsymbol{B}=\nabla \times \boldsymbol{A}$ is then evolved forward in time 
L5
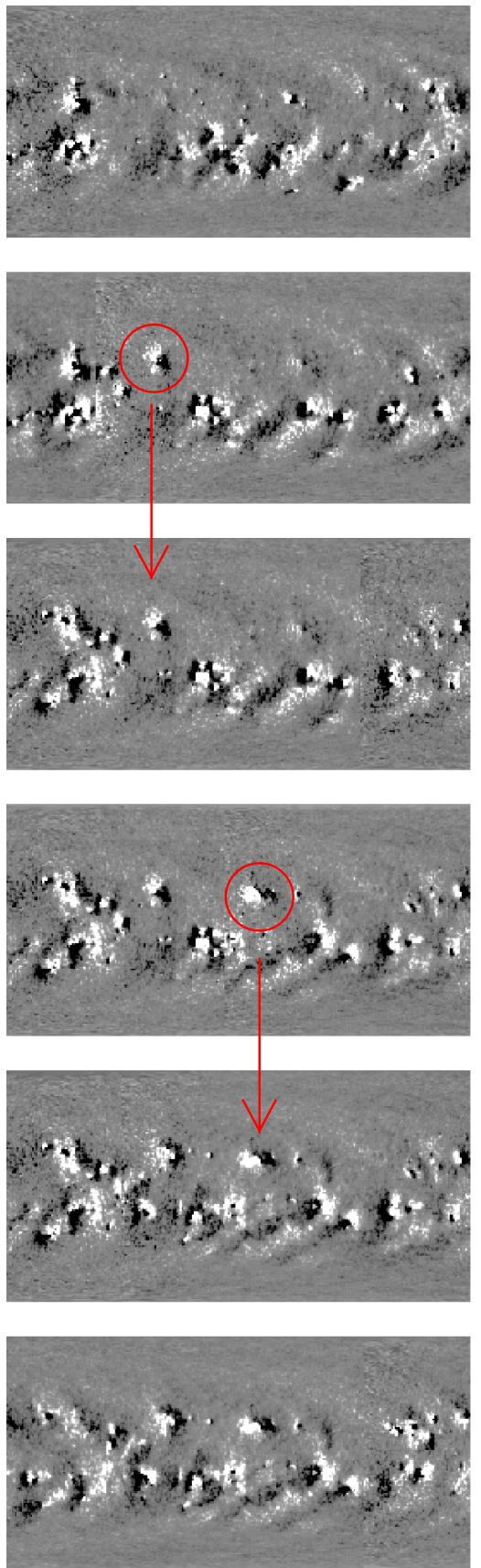

L1
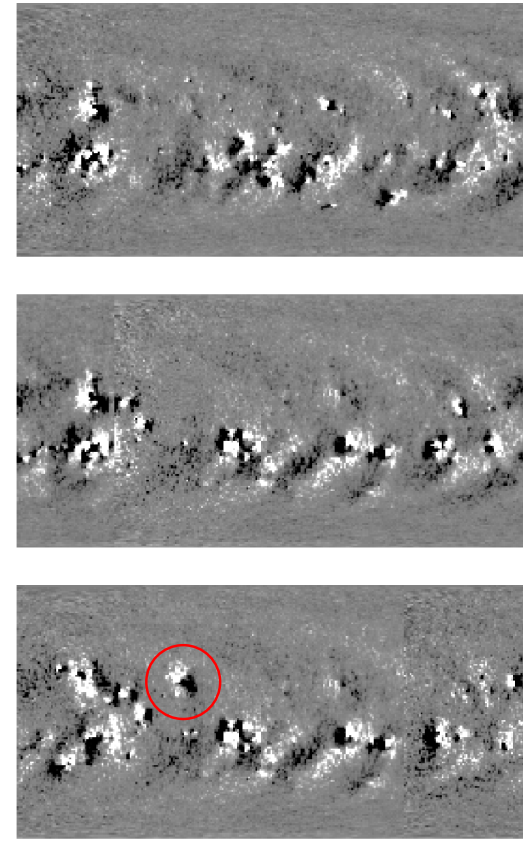

Day

29
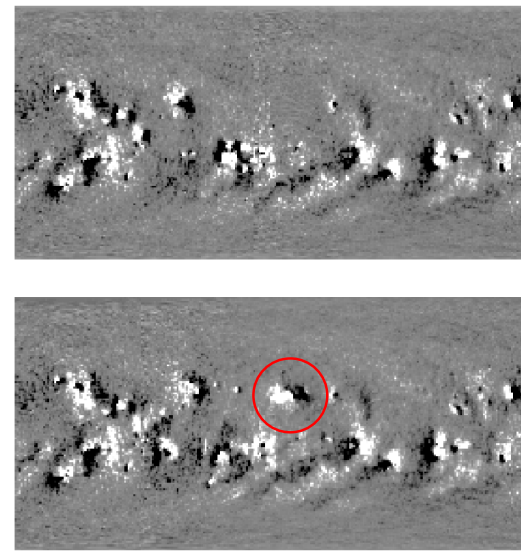

Day 47

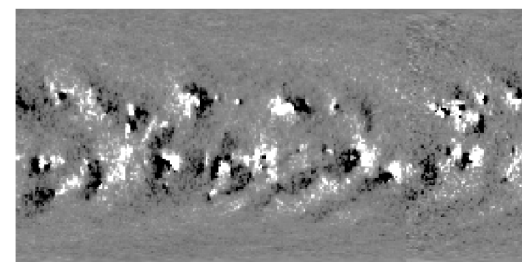

Figure 1. Radial magnetic field at the solar surface in the two data sets produced from observed synoptic data for L5 (left column) and L1 (right column) on selected days. The red circles highlight the newly visible active regions whose appearance in L1 has been delayed. The arrows indicate the time frame until the active regions are observed in L1. In each image, the rotation of the Sun has been removed, and white/black represents positive/negative flux (saturated at $\pm 50 \mathrm{G}$ ).

using the uncurled form of the magnetic induction equation,

$$
\frac{\partial \boldsymbol{A}}{\partial t}=\boldsymbol{v} \times \boldsymbol{B}-\eta \nabla \times \boldsymbol{B},
$$

with diffusion coefficient $\eta$. The use of the magneto-frictional technique enforces the relaxation of the magnetic field toward a nonlinear force-free state where $\boldsymbol{J} \times \boldsymbol{B}=0$. The MF model allows for a gradual build-up and conservation of magnetic energy and electric currents in the corona in contrast to common extrapolation methods, which have no memory of magnetic connectivity.
The simulations in this paper use a fixed, regular spherical grid $R_{\odot} \leqslant r \leqslant 2.5 R_{\odot}, 8.3 \leqslant \theta \leqslant 171.7$ (i.e., the poles are cut off), $0^{\circ} \leqslant \phi \leqslant 360^{\circ}$, with grid cells equally spaced in stretched coordinates (van Ballegooijen et al. 2000), with 28 cells in radius, 160 cells in latitude, and 192 cells in longitude. At the latitudinal boundaries $\left(\theta=8^{\circ} .3\right.$ and $\left.\theta=171^{\circ} .7\right)$, we impose $B_{\theta}=v_{\theta}=0$. The inner boundary conditions (at $r=R_{\odot}$ ) are taken from a sequence of line-of-sight magnetograms, as described in Sections 2 and 3.2. At the outer boundary $\left(r=2.5 R_{\odot}\right)$, we impose zero radial gradient in $B_{\theta}$ and $B_{\phi}$, along with a radial outflow. This ensures that $\boldsymbol{B}$ remains predominantly radial near this boundary, while 


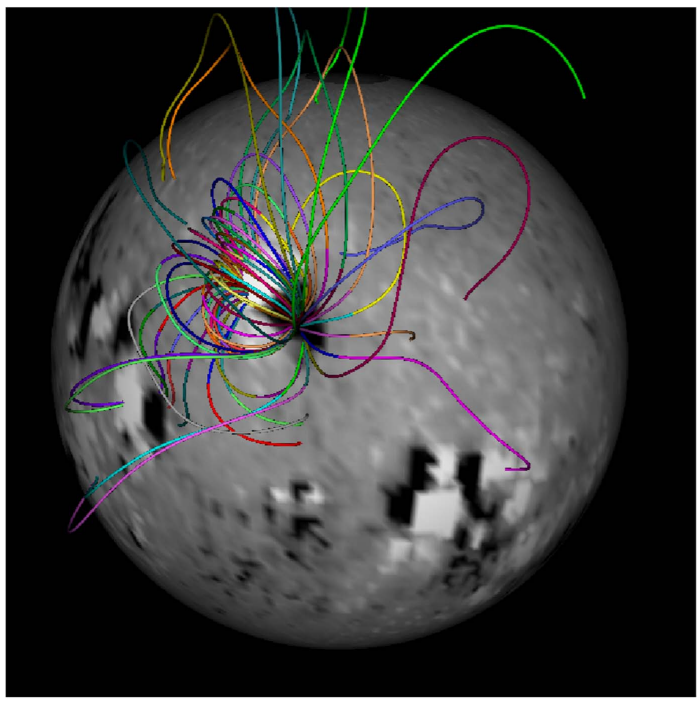

(a) L1, Day 21. $\left[\mathbf{E}_{\text {pot }}, \mathbf{E}_{\text {non-pot }}, \mathbf{E}_{\text {free }}\right]=$ $[8.31,9.14,0.83] \times 10^{33}$ erg.

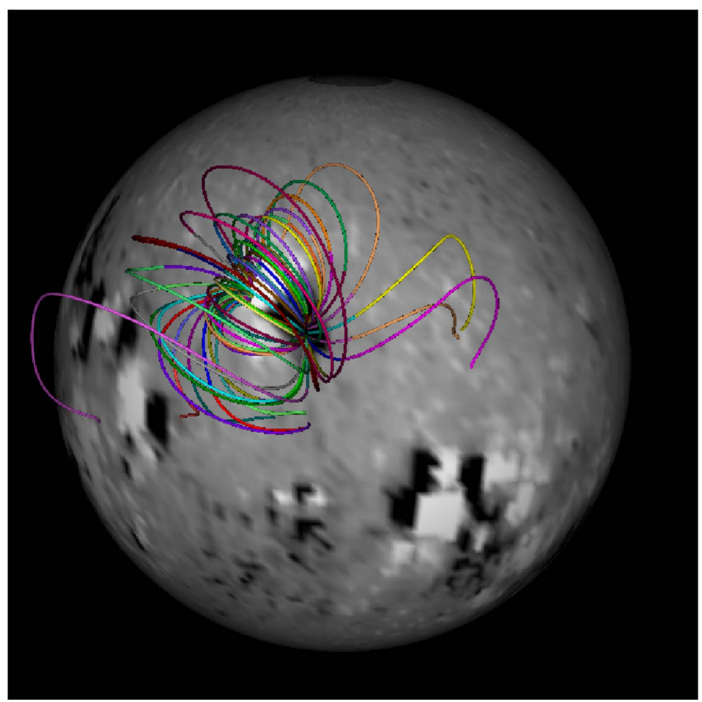

(c) L5, Day 21. [ $\left.\mathbf{E}_{\text {pot }}, \mathbf{E}_{\text {non-pot }}, \mathbf{E}_{\text {free }}\right]=$ $[8.31,9.08,0.77] \times 10^{33} \mathbf{e r g}$.

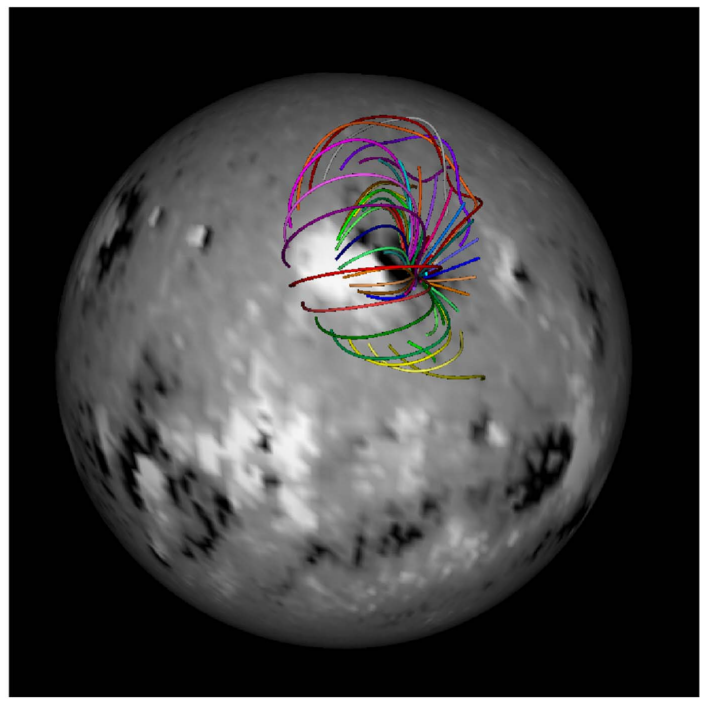

(b) L1, Day 36. $\left[\mathbf{E}_{\text {pot }}, \mathbf{E}_{\text {non-pot }}, \mathbf{E}_{\text {free }}\right]=$ $[7.73,8.73,1.00] \times 10^{33} \mathrm{erg}$.

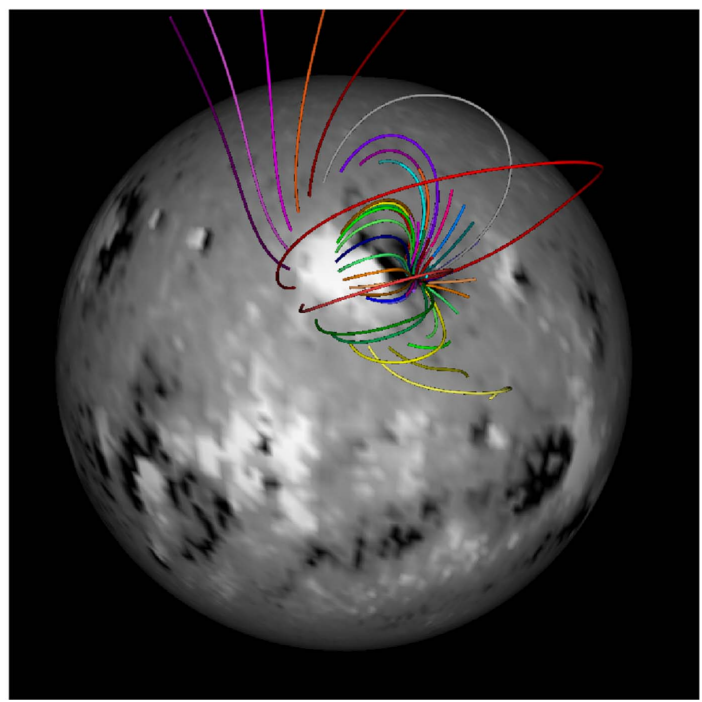

(d) L5, Day 36. [ $\left.\mathbf{E}_{\text {pot }}, \mathbf{E}_{\text {non-pot }}, \mathbf{E}_{\text {free }}\right]=$ $[7.73,8.65,0.92] \times 10^{33}$ erg.

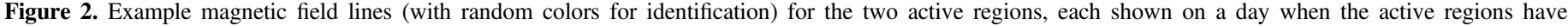

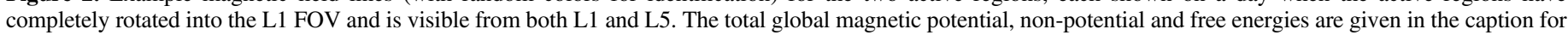
each case.

allowing flux ropes to be ejected (see also Mackay \& van Ballegooijen 2006; Yeates et al. 2010). The coefficient of coronal diffusion is set to be $\eta=60 \mathrm{~km}^{2} \mathrm{~s}^{-1}$.

\subsection{Photospheric Boundary Conditions}

Unlike Mackay et al. (2016), who drove the MF simulation with a surface flux transport model on the photospheric boundary, here we use the data-driven technique developed by Weinzierl et al. (2016). This technique deduces the horizontal electric field $\boldsymbol{E}_{\perp}$ solely from line-of-sight magnetograms $B_{r}$, without using additional observational data. It is a special case of the Poloidal-toroidal Decomposition-Doppler-Fourier Local Correlation Tracking-Ideal (PDFI) approach (see also Fisher et al. 2010, 2012; Kazachenko et al. 2014, 2015; Fisher et al. 2015) where Doppler contributions are set to zero and the horizontal velocity is determined from the differential rotation profile rather then from correlation tracking. The horizontal electric field $\boldsymbol{E}_{\perp}$ is needed to evolve the magnetic field $\boldsymbol{B}=\nabla \times \boldsymbol{A}$ by providing an update $\partial \boldsymbol{A} / \partial t=-\boldsymbol{E}$ to the vector potential $\boldsymbol{A}$. It can be written as

$$
\boldsymbol{E}_{\perp}=-\nabla \times\left(\frac{\partial \Phi}{\partial t} \boldsymbol{e}_{r}\right)-\nabla_{\perp} \frac{\partial \psi}{\partial t},
$$



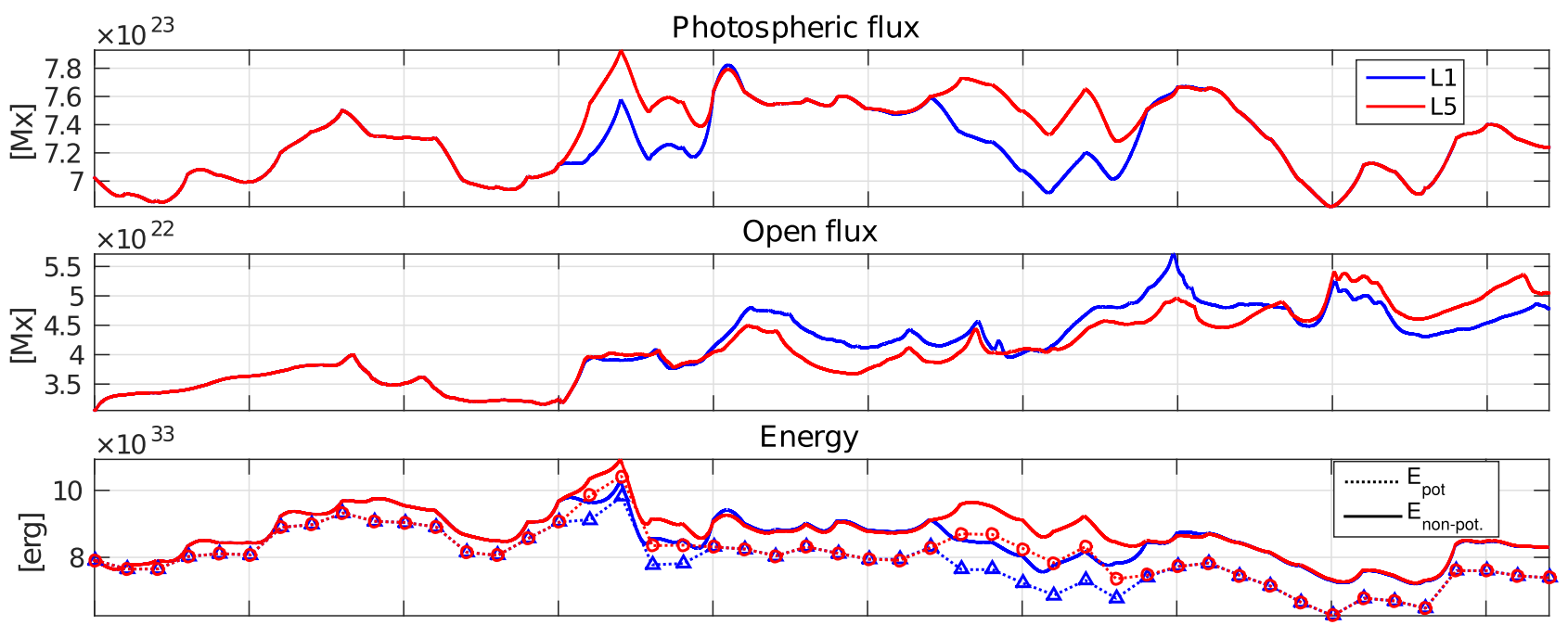

Average $|j|$

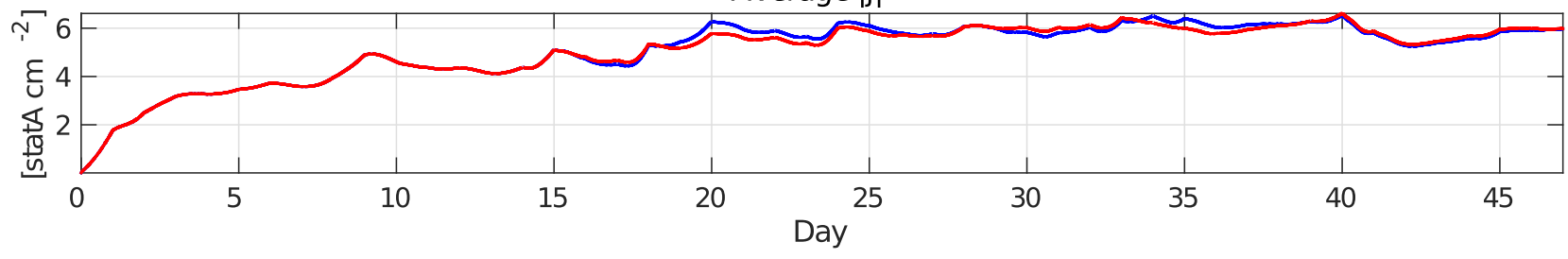

Figure 3. Global integrated quantities for the L1 (blue) and L5 (red) simulations. The top panel shows the total unsigned magnetic flux through the photosphere; the second panel shows the total unsigned open magnetic flux (i.e., through the outer boundary); the third panel shows the total non-potential magnetic energy (solid lines) and potential field energy (triangles for L1 and circles for L5); and the fourth panel shows the averaged electric current $|j|$ in the volume.

(e.g., Amari et al. 2003; Fisher et al. 2010; Kazachenko et al. 2014). The first term, the so-called "inductive part," can be uniquely derived from a sequence of $B_{r}$ maps by solving

$$
\nabla_{\perp}^{2} \frac{\partial \Phi}{\partial t}=-\frac{\partial B_{r}}{\partial t}
$$

which is derived from Faraday's law of induction. The second term in Equation (3) includes the unspecified scalar potential $\psi$, which cannot be computed from $B_{r}$ alone and is not uniquely defined without additional information. Rather than setting $\partial \psi /$ $\partial t=0$, as done, for example, in Mackay et al. (2011) and Gibb et al. (2014), we follow Weinzierl et al. (2016) and solve

$$
\nabla_{\perp}^{2} \frac{\partial \psi}{\partial t}=\nabla \cdot\left(v_{\phi} B_{r} \boldsymbol{e}_{\theta}\right)
$$

This is derived from ideal Ohm's law, and accounts for the contribution to $\partial \psi / \partial t$ from a known differential rotation profile $v_{\phi}$.

\section{GLOBAL PROPERTIES}

\subsection{Integrated Quantities}

Figure 3 shows some of integrated global quantities for the L1 (blue line) and L5 (red line) simulations. These include the photospheric magnetic flux (top panel), the open magnetic flux (second panel), the magnetic energy (third panel; the solid lines for non-potential simulations and the dashed lines with symbols for potential extrapolations), and finally the volume-integrated electric current (bottom panel).

The photospheric flux is identical in both the L1 and L5 simulations until day 15 , when the first active region emerges in L5 but is not seen in L1. This leads to increased flux in L5 until the active region rotates into the L1 view on day 20 . Between days 15 and 20, both L1 and L5 show similar variations and vary in phase with one another, reflecting the fact that only the region around this active region is changed in the L1 data set. After day 20, the photospheric flux again matches in both simulations, until the second active region emerges on day 27 in L5 and the process repeats itself. This illustrates quantitatively the features that were described in Section 2, namely, that the only difference between the L1 and L5 data sets is located around the position of emergence of the two active regions. At other locations the photospheric pattern of radial magnetic field is identical.

In both the L1 and L5 simulations, a similar behavior to that seen for the surface flux is found for the volume-integrated magnetic energy. In the third panel, the solid lines denote the results for the two non-potential simulations, whereas the dotted lines with symbols (circles for L5 and triangles for L1) show the energy of the corresponding potential field that matches the same radial field at the photosphere in each simulation. Once again, the simulations match each other during the times where the photospheric fields are identical, but during the times where the active regions exist in L5 but not in L1, the L5 simulation has higher energy (both potential and non-potential). This difference can be as large as $1 \times 10^{33} \mathrm{erg}$; in comparison, the largest solar flares release about $6 \times 10^{32}$ erg. In both the L5 and L1 simulations, the free magnetic energy (i.e., the difference between the non-potential and potential energies) can also be around $1 \times 10^{33} \mathrm{erg}$. While the L5 simulation overall has a higher energy, it is interesting to note that the free magnetic energy of the field can be slightly higher in the L1 simulation, during periods where the active region exists in L5 but not in L1. This is an interesting feature whose origin will be discussed when we consider the evolution 
of current helicity later in the paper. It does, however, illustrate that more magnetic flux does not necessarily mean more free energy to power eruptions. This is a consequence of the fact that when the active region initially emerges in L5 it is in a state that is close to potential. It dominates over and breaks up any previous non-potential fields that existed at its location. These non-potential fields, however, still (incorrectly) exist in the L1 case.

There are no significant differences in the variation and levels of the total electric current (bottom panel of Figure 3, less than $8 \%$ maximum difference) between the two simulations, although significant differences do occur in the variation of the open flux (second panel). For the open flux, up until day 15 , the two simulations follow an identical evolution. However, after this date they start to diverge as a consequence of the emergence of the first active region in the L5 simulation. The two curves do not come back together again, even after the active regions are present in both simulations. While the absolute levels are not the same, both simulations do show the same features that vary in phase with one another. As they follow the same behavior we conclude that, at least for this data example, there are no missed or delayed flux rope ejections in the L1 simulation, relative to L5. This expectation is confirmed when looking at the 3D magnetic field and flux ropes.

One interesting feature of the open flux seen in both the L1 and L5 simulations between days 40 and 43 is the occurrence of an oscillation. This is a signature of the eruption of two flux ropes which lie in close proximity to one another. One erupts slightly before the other, with both ejected out of the box. This give two enhancements of the open flux in close succession and the appearance of an oscillation.

From Figure 3, it is clear that two different types of behavior are found in key global quantities as a result of the local differences between the two photospheric data sets. Surface flux and magnetic energy differ only during the time period when the photospheric magnetic field differs, after which the curves come together again. Thus, while missing local information in the L1 FOV leads to underestimating the total energy, this error is not retained in the corona over long periods of time. In contrast, differences are retained for longer periods in the open flux, with the L1 non-potential simulation no longer producing the correct level of open flux as in L5. This indicates that missing a local feature in the input magnetograms for only a few days, even if the magnetograms are everywhere else identical, can lead to a long-term global difference in the nonpotential model. This is extremely important as the open flux is one critical component used to drive the present generation of space weather prediction models.

\subsection{Current Helicity Density}

To consider what effect the local difference in appearance time of the active regions has on the global corona, we now consider the current helicity distributions in the simulation. Figure 4 shows plots of the current helicity density

$$
\alpha=\frac{\boldsymbol{B} \cdot \boldsymbol{J}}{B^{2}}=\frac{\boldsymbol{B} \cdot(\nabla \times \boldsymbol{B})}{B^{2}}
$$

at a height of $0.03 R \odot$ above the photosphere, at key times during the emergence of the active region in L5 and its subsequent appearance in L1. In the MF model, where the field is approximately force-free (so that $\boldsymbol{J} \approx \alpha \boldsymbol{B}$ ), this quantity illustrates the location of electric current and thus the non- potential nature of the coronal field. The top row shows the results for $\mathrm{L} 1$ on days 17,21 , and 27 , while the middle row shows the same days for L5. In both cases, the red/blue represent positive/negative $\alpha$. Finally, the bottom row illustrates the difference between the two simulations using the same color scale.

The first column of Figure 4 considers the $\alpha$ value on day 17, when the active region has emerged in L5 but is not yet visible in L1. In both the L1 and L5 simulations, it is clear that there is a wide range of $\alpha$ values and that non-potential fields cover all longitudes and latitudes on the Sun. In addition, regions with both signs of $\alpha$ exist at all longitudes and latitudes across the surface. A careful comparison of Figures 4(a) and (d) shows that at the location where the first active region has emerged in L5, there are small $\alpha$ values (denoted by white), whereas the L1 simulation has a strong mixing of positive and negative $\alpha$. This demonstrates that as the active region emerges in L5, it does so in a state close to potential. This is expected, because our noninductive electric field component $\partial \psi / \partial t$ does not include a contribution representing the emergence of twisted magnetic field in the active region (see also Cheung \& DeRosa 2012). This potentiality is a contributing factor to the lower free energy at this time in the L5 simulation compared with L1, even though the surface flux is much greater. The result here is also consistent with the results of Régnier \& Priest (2007), who found that new active regions are in a state that is closer to potential than older regions. It also illustrates that knowledge of only the complexity and strength of the photospheric field is not necessarily a good indicator of whether an eruption can take place. To determine if and what size of an eruption can take place, knowledge of the coronal field is required.

From Figure $4(\mathrm{~g})$ it is interesting to note that the local emergence of the active region in L5 (with the same photospheric field everywhere else) not only influences the current helicity at this location, but also over the whole domain. The most significant differences can be seen to occur in the northern hemisphere, localized around the active region, but differences do occur across all longitudes, and in both hemispheres. It is also interesting to note that the size of the differences can be comparable to the magnitude of $\alpha$ itself, and can include differences in sign.

On day 21 (middle column Figure 4), when the active region is visible in both simulations, significant differences can still be seen between the simulations. Figure 4(h) shows that the strongest differences in $\alpha$ are again located at the position of the active region. In addition, in the northern hemisphere the differences extend across a much wider longitudinal range than in the southern hemisphere. By day 27 (the right-hand column in Figure 4), many of the differences are now decreasing and both simulations have begun to relax back to a similar state.

To further illustrate the behavior of $\alpha$, Figure 5 shows longitudinal averages of $\alpha$ at six times, covering both active region emergences. In each plot, blue represents the results from L1 and red L5, while the longitudinal average of their difference is shown in green. The left-hand column shows days around the first active region emergence, while the right-hand column shows days around the second. In each column, the top row shows dates where the active region has emerged in L5 but not in L1. For these days, there are significant differences for the first active region emergence, with greatest differences in the northern hemisphere above $40^{\circ}$ latitude. Smaller differences are found for the second active region emergence, although for 


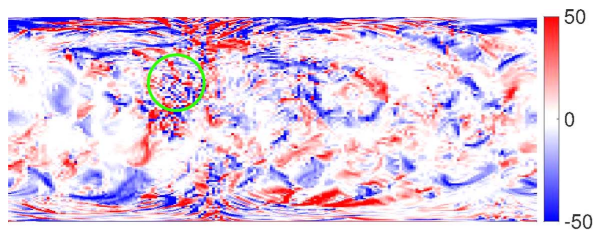

(a) L1, Day 17

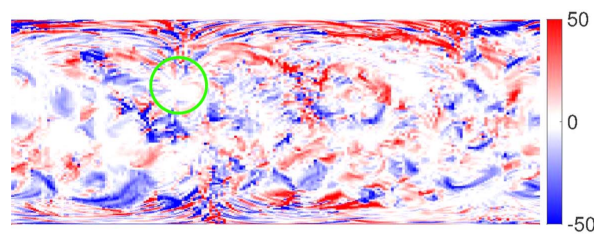

(d) L5, Day 17

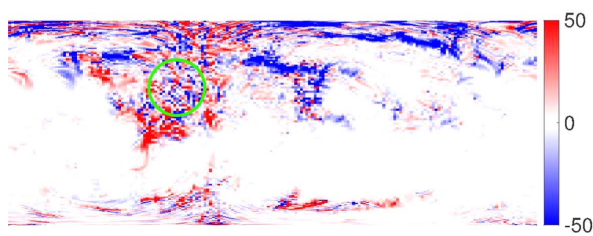

(g) Difference, Day 17

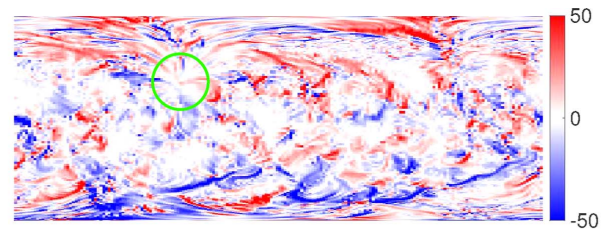

(b) L1, Day 21

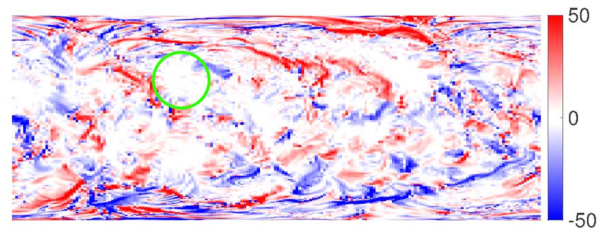

(e) L5, Day 21

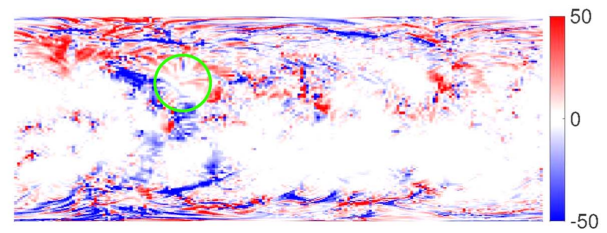

(h) Difference, Day 21

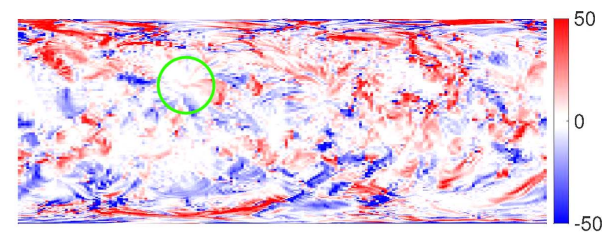

(c) L1, Day 27

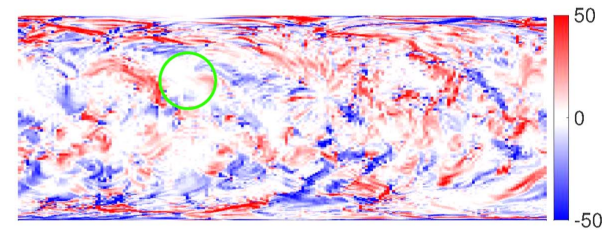

(f) L5, Day 27

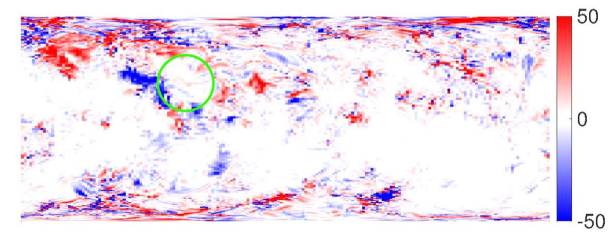

(i) Difference, Day 27

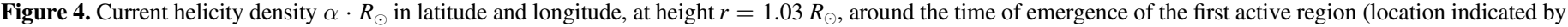
the green circle). The top and middle rows show the results for the L1 and L5 simulations, respectively. The bottom row shows the difference between the two.

both L1 and L5 the absolute values of the average are less, indicating a stronger intermingling of signs of $\alpha$ at all latitudes.

The middle panel considers the days where the active regions have now rotated into the L1 FOV. Even though the active regions are now included in both simulations, there are still significant differences in $\alpha$ in both hemispheres. By the time shown in the bottom row, the active regions have had a significant chance to evolve and interact with their surroundings in both simulations. After this, it is clear that the differences are lower, with a good agreement over a wide range of latitudes. The majority of differences that remain are at the poles.

\subsection{Open Magnetic Flux}

For space weather purposes, one of the most important outputs is the open magnetic flux. It is key for understanding the solar wind and as an input into space weather prediction models such as Enlil (Odstrčil \& Pizzo 1999). We have already shown in Figure 3 that significant differences occur in the amount of open flux between the L1 and L5 simulations. We now consider the reasons for this by comparing the spatial distribution of open magnetic field between the two simulations. Locations of the open field footpoints on the solar surface are shown in Figure 6.

For the duration of the first active region emergence, over days $17-27$, it can be seen that a generally similar pattern of the open flux is found in both simulations. There are, however, significant differences, both at longitudes associated with the active regions but also at other locations. The most distinct differences are: (i) Days 17-27 at the location of the active region around $\phi=120^{\circ}$, $\theta=40^{\circ}$. (ii) Day 21 at the equator around $\phi=180^{\circ}$ and (iii) Day 27 in the northern hemisphere between $\phi=300^{\circ}-360^{\circ}$ and $\theta=40^{\circ}-60^{\circ}$. It is interesting to note that local active region emergences at different times in L5 and L1 can lead to global differences in the open field line footpoints that may be far from the physical location of the active region. The reason for this is that when the active regions emerge at different times they interact with different overlying coronal fields. Thus, even though the photospheric fields in L1 after emergence are identical to that in L5, the connectivity of the active region is different. As the simulations of the non-potential field conserve flux and magnetic connectivity of the field lines different interaction and open flux propagates through the simulations. Similar features can also be found for the period covering the second active region emergence (Figures 6(j)-(r)).

\section{LOCAL PROPERTIES}

In the previous section, we considered the global effect on the corona when the two active regions are included at different times in the L5 and L1 simulations. In this section, we focus on the local differences that occur within the active regions themselves.

Figure 7 shows the magnetic connectivity of the active region that emerges on day 15 in the L5 simulation and then rotates into the L1 FOV on day 20. The images illustrate the connectivity of the coronal magnetic field on day 22 (Figures 7(a)-(c)), shortly after the active region has appeared in L1 as well as L5. The second row (Figures 7(d)-(f)) shows the active region on day 27 after it has had time to interact with the surrounding field in both the L1 and L5 simulations. Figures 7(a) and (d) show the results from L5, while (b) and (e) show the results from L1. Finally, Figures 7(c) and (f) illustrate the differences in connectivity. For the first two figures in each row, the red lines represent field lines plotted from the positive polarity of the active region, while the blue lines denote field lines plotted from the negative polarity. To trace the field lines, nine field lines are traced from different positions within every pixel where $B_{r}>50 \mathrm{G}$. The same starting locations are used in both the L1 and L5 simulations. From considering the field lines, it can be seen that on both day 22 and day 27 the field lines that 


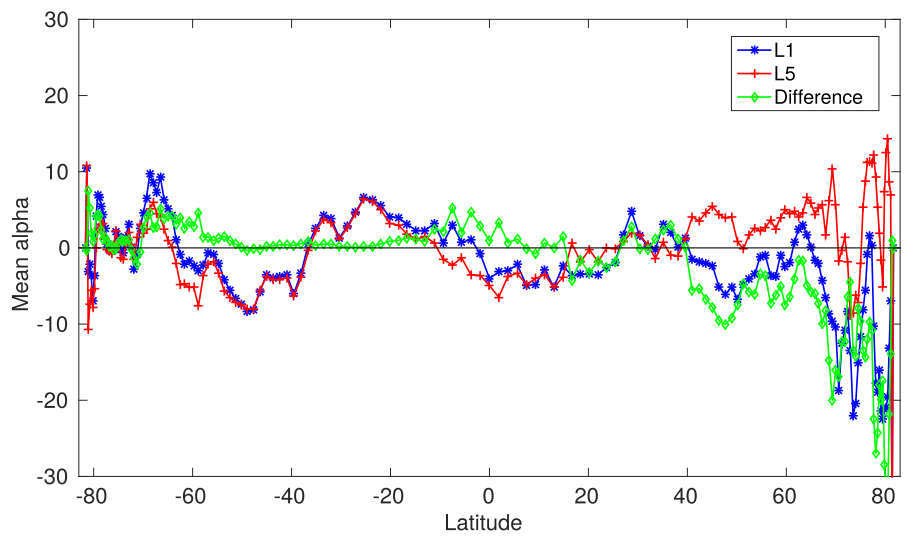

(a) Day 17

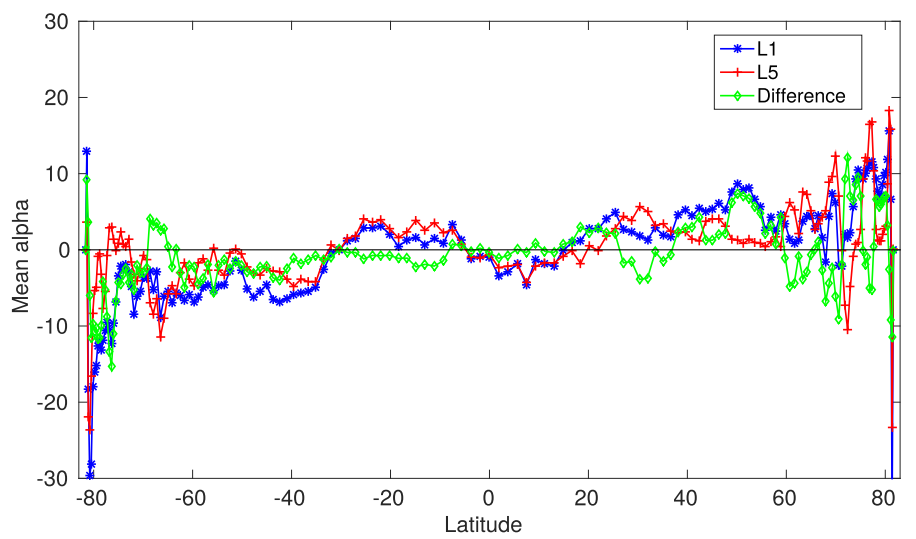

(c) Day 21

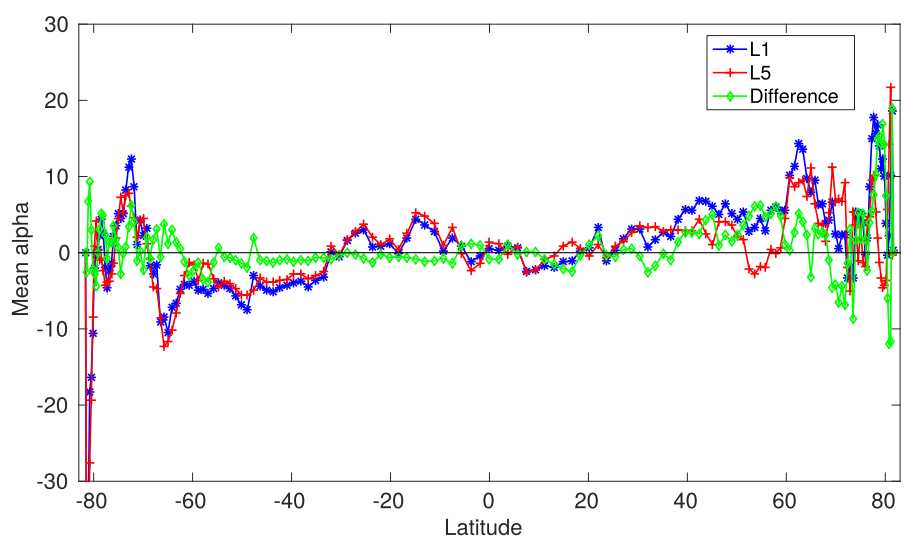

(e) Day 27

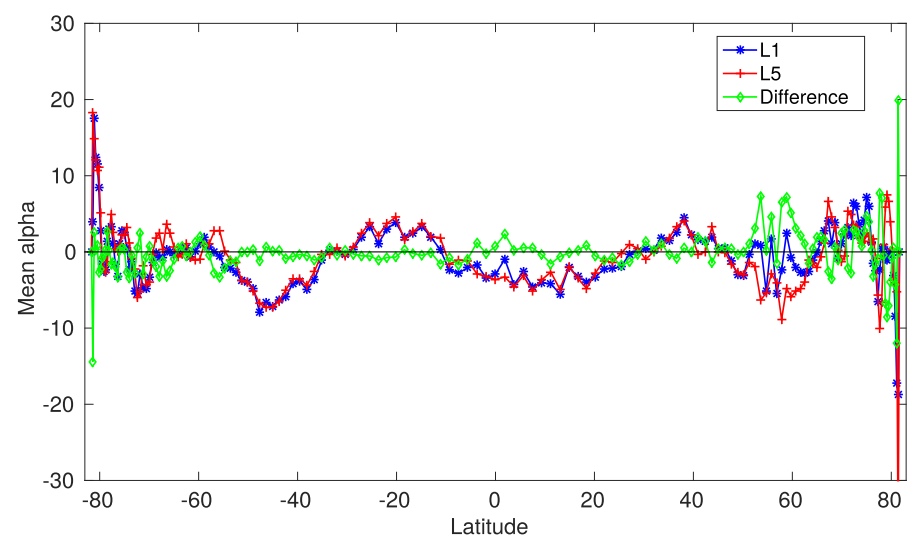

(b) Day 29

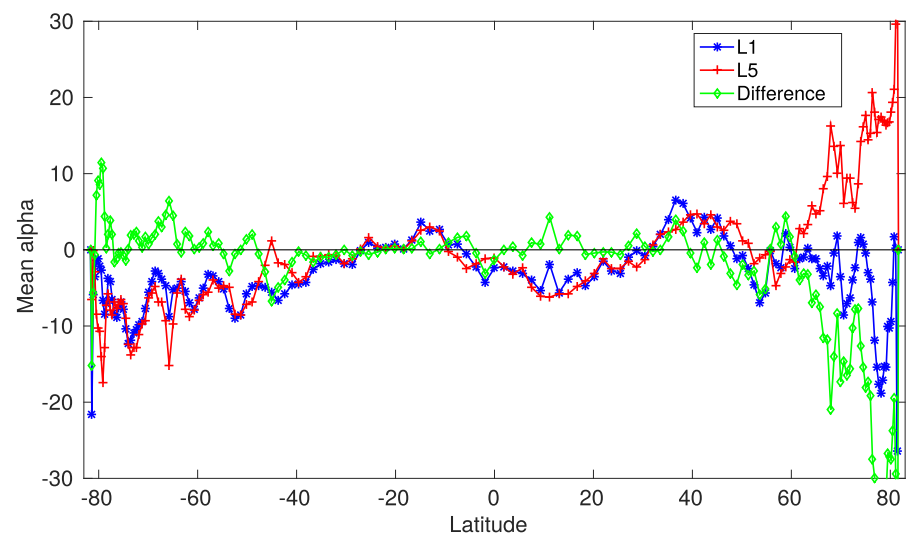

(d) Day 36

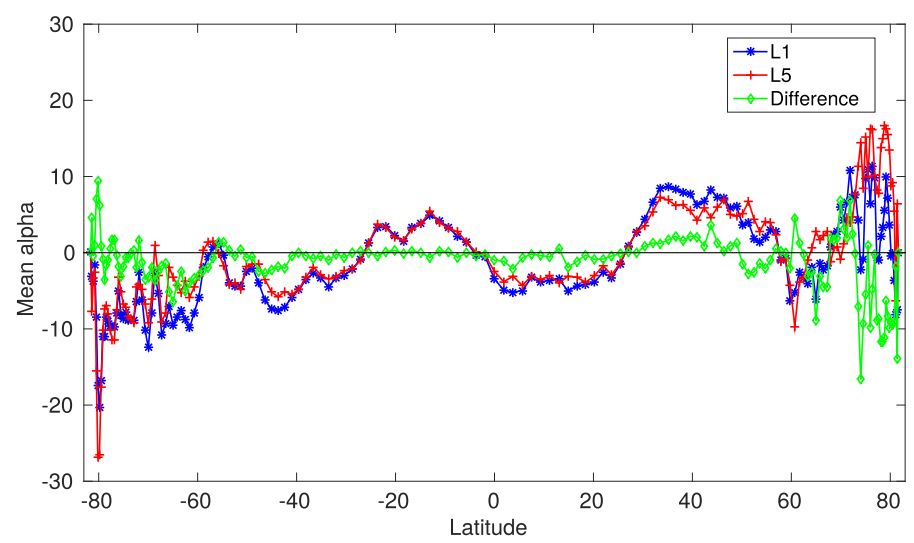

(f) Day 43

Figure 5. Longitude-average of current helicity density $\alpha$ at height $r=1.03 R_{\odot}$ around the time of emergence of the first (left) and second (right) active regions. The top row shows the time when the active regions appear in the L5 simulation, the middle row is the time when they are seen (completely) from L1, and the bottom row is some days later.

connect between the positive and negative polarities of the active region appear very similar in both simulations. In contrast, the field lines that connect the active region to its surroundings are different. In particular, the positive polarity of the active region has an east-west connectivity toward lower longitudes in L5, while in L1 the connection is more north-south. Also, on day 27 it is clear that in the L5 simulation no field lines connect out of the image toward the top right, whereas in the L1 simulation this connectivity can be clearly seen.

To quantify these differences in field-line connectivity, the right-hand column in Figure 7 shows the differences in the endpoints of field lines between the L1 and L5 simulations, traced from the same starting points in both simulations. The starting points are color-coded according to the spatial extent of the endpoint difference, according to the following.

1. Green: the endpoints lie within the same pixel (of the photospheric magnetogram).

2. Blue: the endpoints differ by more than one pixel but less than two.

3. Yellow: the endpoints differ by more than two pixels.

4. Red: the field line is closed in one simulation but open in the other. 


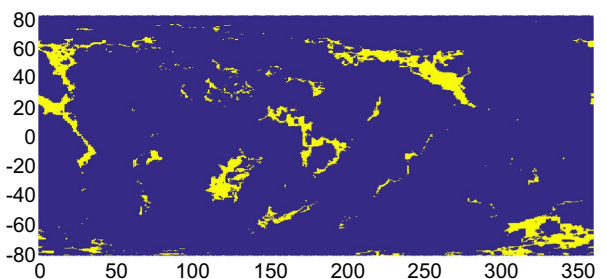

(a) L1, Day 17

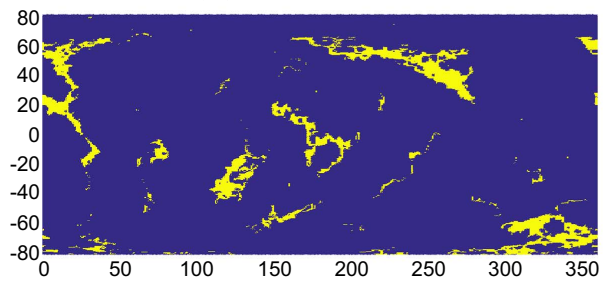

(d) L5, Day 17

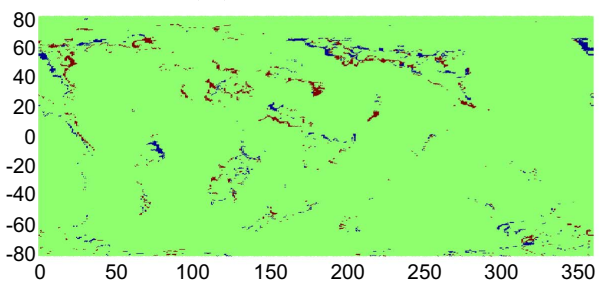

(g) Difference, Day 17

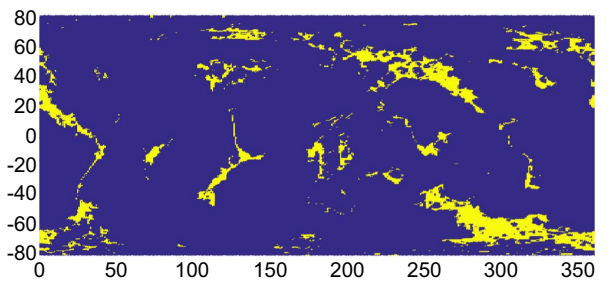

(j) L1, Day 29

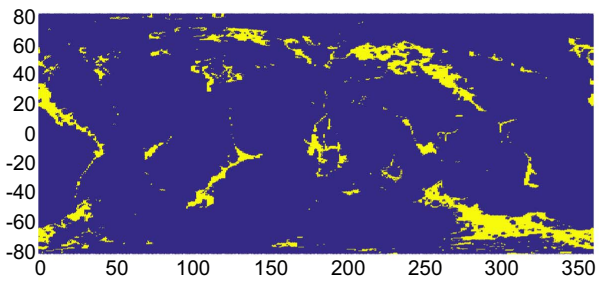

(m) L5, Day 29

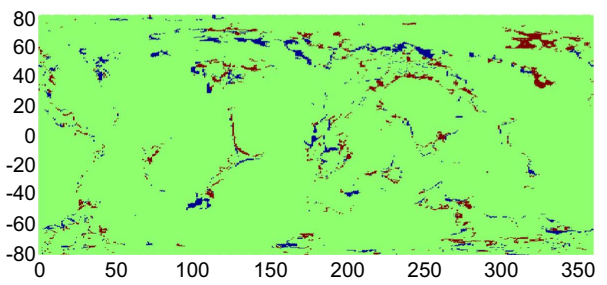

(p) Difference, Day 29

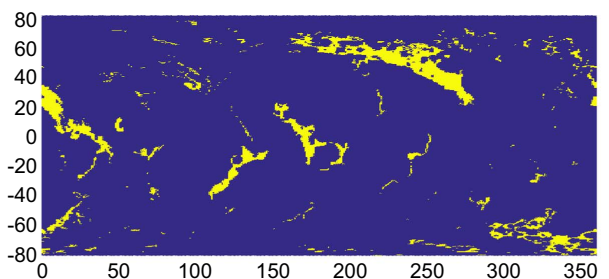

(b) L1, Day 21

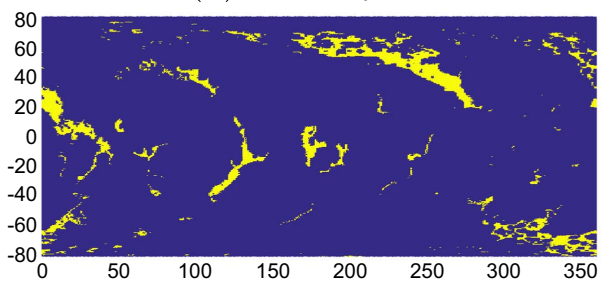

(e) L5, Day 21

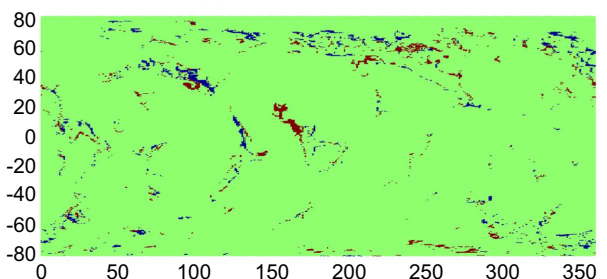

(h) Difference, Day 21

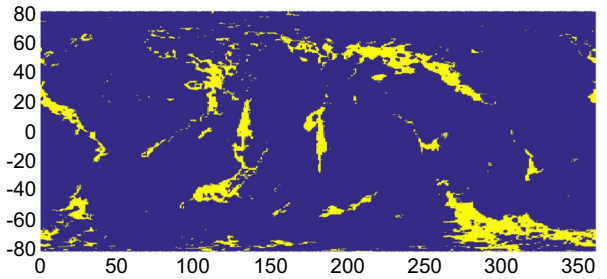

(k) L1, Day 36

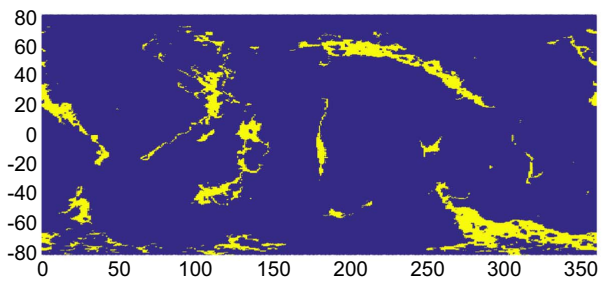

(n) L5, Day 36

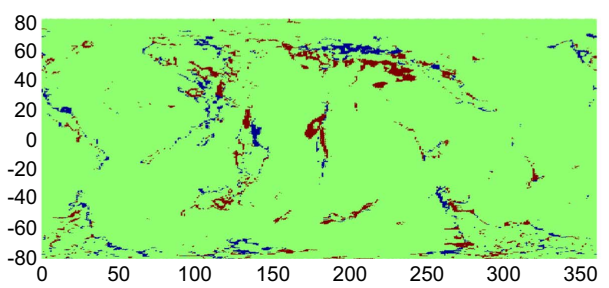

(q) Difference, Day 36

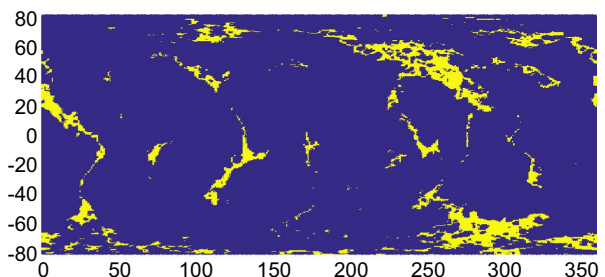

(c) L1, Day 27

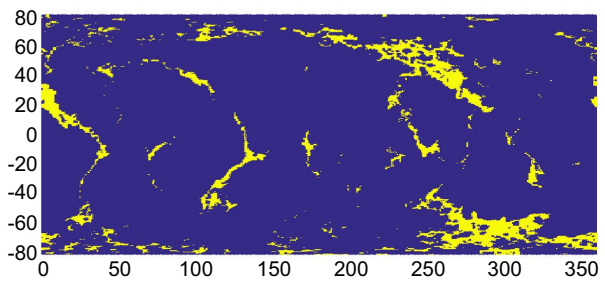

(f) L5, Day 27

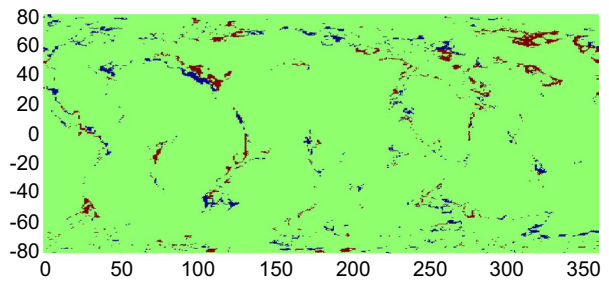

(i) Difference, Day 27

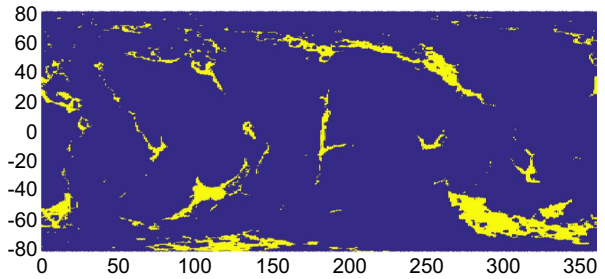

(1) L1, Day 43

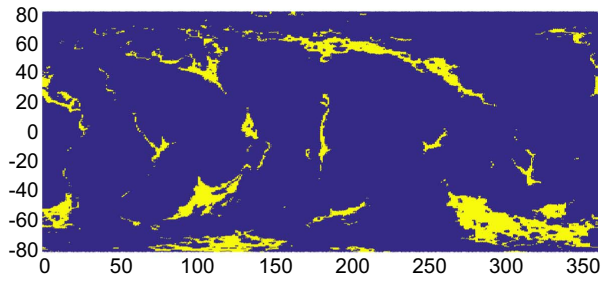

(o) L5, Day 43

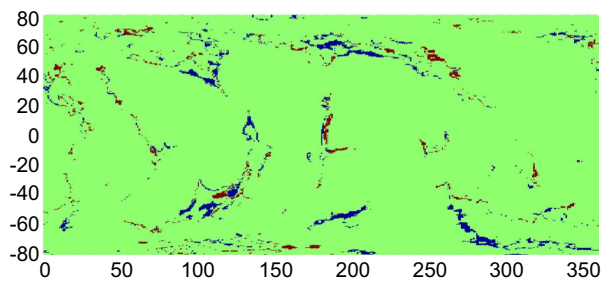

(r) Difference, Day 43

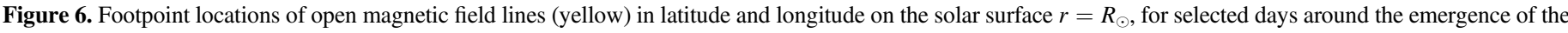

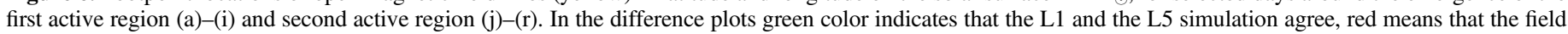
line is open in L1 and closed in L5, and blue that it is open in L5 and closed in L1.

From this color-coding, it can be seen that although the active regions emerge at different times in the two simulations, the central parts of each active region have essentially the same connectivity (green or blue) on days 22 and 27. Where significant differences do occur (red or yellow), they are around the outer edges of the active regions. These differences in connectivity can be clearly seen on comparing Figures 7(a) and (b) or (d) and (e). Also, there are a number of locations where 


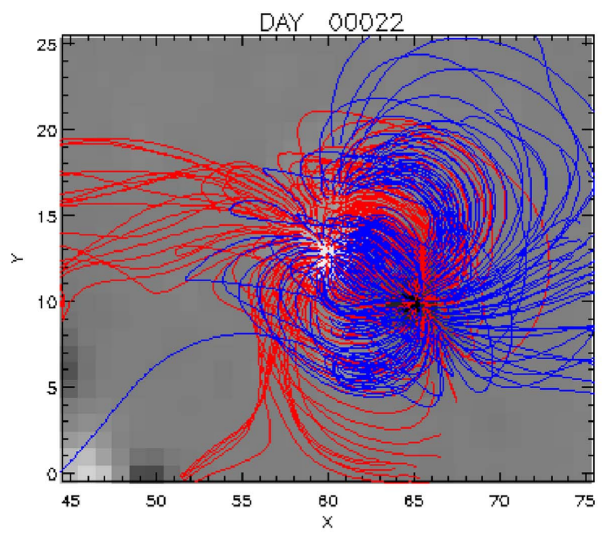

(a) L5

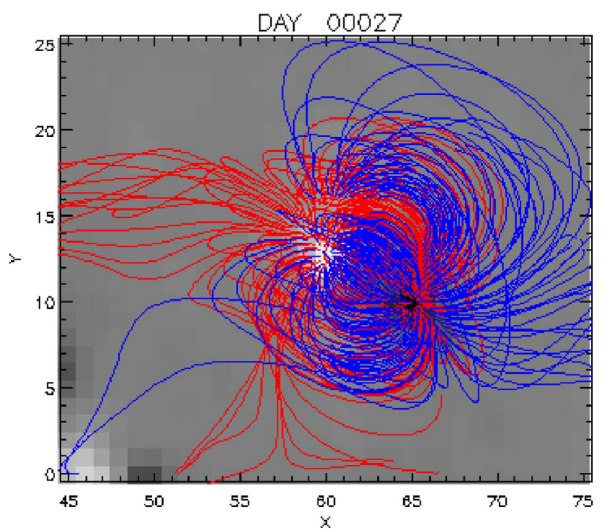

(d) L5

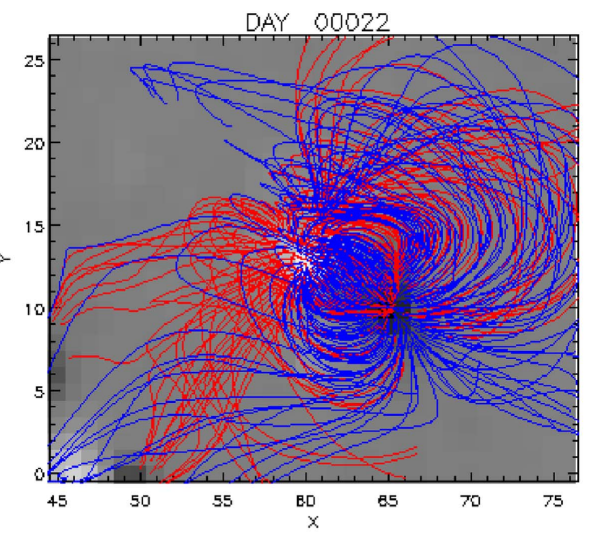

(b) L1

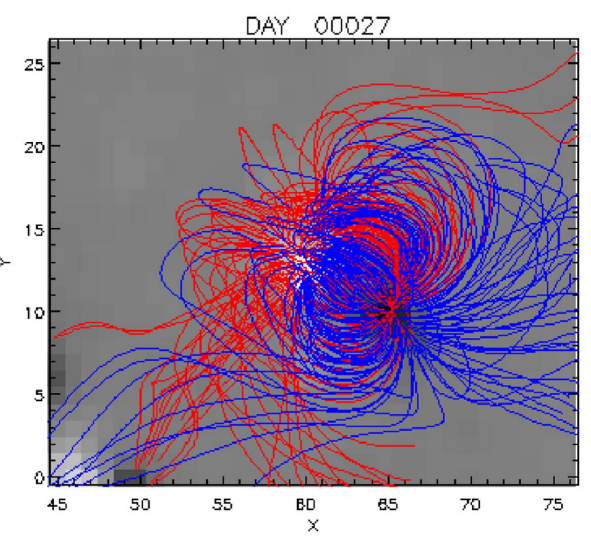

(e) L1

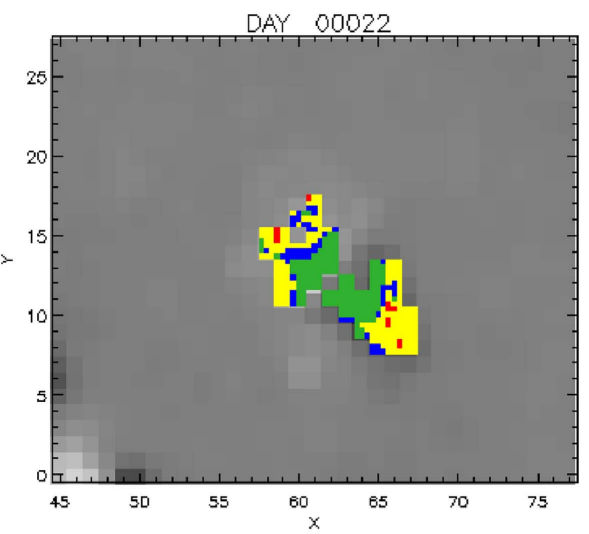

(c)

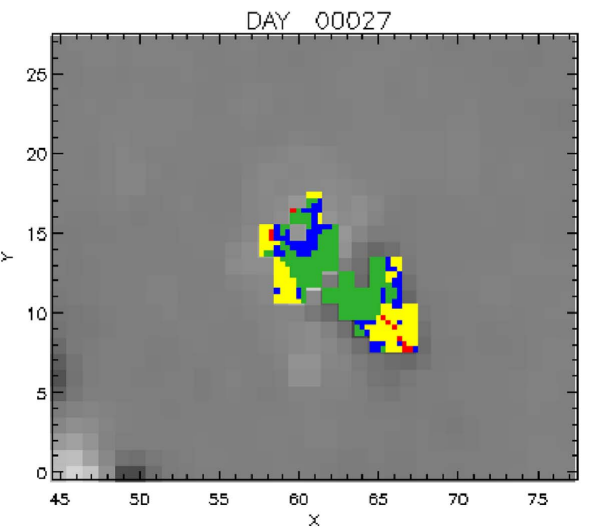

(f)

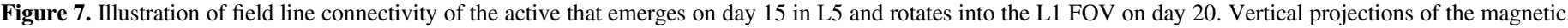

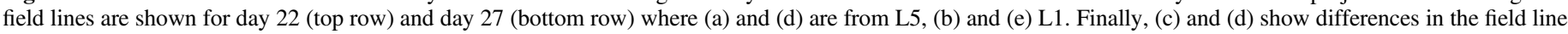
connectivity (the colors are explained in the text). In each case, red/blue field lines are traced from the positive/negative polarity.

field lines are open in one simulation and closed in the other, illustrating that the open flux and solar wind would be originating from different locations within the active region in each simulation. While many differences in connectivity occur on both day 22 and day 27, the differences are tending to decrease by day 27 , as can be seen from the increased areas of blue and green and decreased areas of yellow. From this, it is clear that missing part of the active regions evolution has a significant effect at the early stages, although these difference become less with time. However, although the differences within the active region decrease, the long-range connectivity of the active region in L1 is still significantly different from that in L5, even at day 27. Such differences would not be seen in potential field modeling or using other extrapolation techniques, but exist in our simulations because we preserve the evolution of magnetic flux and connectivity from one day to the next. Similar results (not illustrated) are found for the second active region and also for intervening days in the evolution.

\section{CONCLUSION}

To assess the possible improvements that an L5 magnetograph could make to non-potential coronal magnetic field models, we artificially delayed the emergence of two active regions in sequences of observed photospheric radial magnetic maps. This simulates the "real-time" emergence of the active regions when observations are available from the L5 point, along with the delayed observation and inclusion of the same active regions when only L1 (or Earth-based) magnetographs are available. The L5 and L1 sequences of maps were then used to drive non-potential magneto-frictional simulations of the coronal magnetic field, representing the situation with and without an additional magnetograph at L5. For the purposes of the present study we regard the L5 data set as representing the "real" Sun as it captures the full evolution of the active regions.

We find that the wrong non-potential structure of the coronal magnetic field can be produced in the L1 simulation when the active region emergence is delayed by only a few days due to the limited FOV. As a consequence of the wrong time of emergence, there are significant differences in magnetic connectivity. Importantly, the active region does not just have a local effect at its emergence location, but a global effect on the entire corona. This is demonstrated clearly by these particular simulations, since the distribution of the photospheric radial magnetic fields are assumed to be identical everywhere, except between the times where the active regions emerge in L5 and subsequent enter into the L1 FOV. Although differences in the photospheric field are minimized, there are significant differences in the coronal magnetic connectivity and in the distribution of current helicity density $(\alpha)$ across the solar surface. Moreover, the coronal magnetic field differences are not limited to the time periods where the photospheric data differ, but persist throughout the entire 48-day simulation. Such differences would not be seen in potential field or other 
extrapolations that are based only on photospheric magnetograms at a single time. This is because they do not retain a memory of flux connectivity from one time to the next.

Once the new active regions have emerged in both the L1 and L5 simulations, the coronal magnetic fields tend to evolve to become more similar. This can be seen in the graphs of the total energy and longitudinal average of $\alpha$. Indeed, there is no significant difference in the formation and eruption times of magnetic flux ropes in the regions concerned. While the simulations do become more similar, we find that significant differences in the longer-range magnetic connectivity between the active region and its surroundings persist for the duration of the simulation. This is in accordance to Pevtsov \& Acton (2001) and Pevtsov \& Kazachenko (2004), who suggested that the localized emergence of an active region may lead to changes in global connectivity in the corona. Most striking is the fact that delaying the observation of even a single active region for only 5 days can lead to persistent differences in open magnetic flux, and in the location of open magnetic footpoints on the solar surface. Such differences could have a significant impact on forecasts of coronal holes, the ambient solar wind, and/or the interplanetary magnetic field (Balogh et al. 1995; Wang \& Sheeley 2002; Hollweg 2008; Cranmer 2009; Lockwood et al. 2009). This indicates that the possible benefit of an L5 magnetograph for space weather prediction could be significant. It also indicates that potential field extrapolations may be unreliable as the basis for solar wind models such as the Wang-Sheeley-Arge model (Wang \& Sheeley 1990a, 1990b, 1992; Arge \& Pizzo 1999, 2000; Arge et al. 2003). Additionally, such long-range connections could be important for triggering so-called sympathetic flares and eruptions (Schrijver et al. 2013; Jin et al. 2016).

While omitting an active region will lead to an underestimate of the total magnetic energy in the corona (at that time), it is not necessarily the case that it will lead to an underestimate of the free magnetic energy. This was found in our examples because the active regions emerge in a state close to potential. It does, however, highlight that knowing only $B_{r}$ on the photosphere is not necessarily a good indicator of whether an eruption can take place from a particular active region. However, we should point out that estimates of the free energy in new active regions are sensitive to the electric field model used to drive the magneto-frictional simulation; here, we neglected additional twisting of the magnetic field during emergence of the region. If such twisting is included, then the free energy in the L5 simulation may become higher than that of L1. In addition, Kazachenko et al. (2014) have shown that in the emergence of an active region, up to $30 \%$ of the actual energy flux can be missed if the Doppler velocity contribution in the PDFI method is neglected. Also, as shown by Longcope et al. (2007), the small-scale dynamics inside an active region (shearing and rotation) can themselves provide enough energy for an eruption. A smaller time cadence of the magnetograms (60-90 minutes) would enable our method to capture some of these motions (Gibb et al. 2014).

In manipulating the magnetogram sequences, we delayed the appearance of only two active regions. In reality, all of the magnetic flux on the surface will be observed later from L1 than from L5. In addition, we neglected evolution of the photospheric magnetic field on the unobserved side of the Sun. Consequently, the differences identified here should be taken as underestimates. Although it is an underestimate, significant differences do occur and this illustrates the importance of L5 observations. Even though the differences in the photospheric field are minimal, the simulations show that even small differences or delays can have a big effect. In previous simulations based on synthetic rather than observational data, Mackay et al. (2016) showed that L5 observations (particularly at active latitudes) could lead to significant improvements in a range of global quantities including open magnetic flux, magnetic energy, and the number of flux ropes present in the corona. Here, we have analyzed the magnetic structure in more detail but over a shorter period of time, both locally and globally, and showed how even delaying the emergence of a single active region in non-potential simulations due to limited observational FOV can have significant global consequences.

This work utilizes SOLIS data obtained by the NSO Integrated Synoptic Program (NISP), managed by the National Solar Observatory, which is operated by the Association of Universities for Research in Astronomy (AURA), Inc. under a cooperative agreement with the National Science Foundation. M.W. and A.R.Y. thank STFC and the US Air Force Office for Scientific Research for financial support. D.H.M. would like to thank STFC and the Leverhulme trust for financial support. M. W., D.H.M., and A.R.Y. additionally thank the International Space Science Institute in Bern for supporting their collaboration.

\section{REFERENCES}

Amari, T., Luciani, J. F., Aly, J. J., Mikic, Z., \& Linker, J. 2003, ApJ, 595,1231

Arge, C. N., Henney, C. J., Hernandez, I. G., et al. 2013, in AIP Conf. Proc. 1539, Solar Wind 13, ed. G. P. Zank (Melville, NY: AIP), 11

Arge, C. N., Henney, C. J., Koller, J., et al. 2010, in AIP Conf. Proc. 1216, Solar Wind 12, ed. M. Maksimovic (Melville, NY: AIP), 343

Arge, C. N., Odstrčil, D., Pizzo, V. J., \& Mayer, L. R. 2003, in AIP Conf. Proc. 679, Solar Wind 10, ed. M. Velli (Melville, NY: AIP), 190

Arge, C. N., \& Pizzo, V. J. 1999, in AIP Conf. Proc. 471, Solar Wind 9, ed. S. T. Suess, G. A. Gary, \& S. F. Nerney (Melville, NY: AIP), 569

Arge, C. N., \& Pizzo, V. J. 2000, JGRA, 105, 10465

Balogh, A., Smith, E. J., Tsurutani, B. T., et al. 1995, Sci, 268, 1007

Cheung, M. C. M., \& DeRosa, M. L. 2012, ApJ, 757, 147

Cranmer, S. R. 2009, LRSP, 6, 3

Fisher, G., Welsch, B., \& Abbett, W. 2012, SoPh, 277, 153

Fisher, G. H., Abbett, W. P., Bercik, D. J., et al. 2015, SpWea, 13, 369

Fisher, G. H., Welsch, B. T., Abbett, W. P., \& Bercik, D. J. 2010, ApJ, 715,242

Gibb, G. P. S., Mackay, D. H., Green, L. M., \& Meyer, K. A. 2014, ApJ, 782,71

Henney, C. J., Toussaint, W. A., White, S. M., \& Arge, C. N. 2012, SpWea, $10, \mathrm{~S} 02011$

Hollweg, J. V. 2008, JApA, 29, 217

Jin, M., Schrijver, C. J., Cheung, M. C. M., et al. 2016, ApJ, 820, 16 Kazachenko, M. D., Fisher, G. H., \& Welsch, B. T. 2014, ApJ, 795, 17 Kazachenko, M. D., Fisher, G. H., Welsch, B. T., Liu, Y., \& Sun, X. 2015, ApJ, 811, 16

Lockwood, M., Rouillard, A. P., \& Finch, I. D. 2009, ApJ, 700, 937

Longcope, D., Beveridge, C., Qiu, J., et al. 2007, SoPh, 244, 45

Mackay, D., \& Yeates, A. 2012, LRSP, 9, 6

Mackay, D. H., Green, L. M., \& van Ballegooijen, A. A. 2011, ApJ, 729, 97

Mackay, D. H., \& van Ballegooijen, A. A. 2006, ApJ, 641, 577

Mackay, D. H., Yeates, A. R., \& Bocquet, F. 2016, ApJ, 825, 131

Odstrčil, D., \& Pizzo, V. J. 1999, JGR, 104, 483

Pevtsov, A. A., \& Acton, L. W. 2001, ApJ, 554, 416

Pevtsov, A. A., \& Kazachenko, M. 2004, in ASP Conf. Proc. 575, SOHO 15

Coronal Heating, ed. R. W. Walsh et al. (San Fransisco, CA: ASP), 241

Régnier, S., \& Priest, E. R. 2007, A\&A, 468, 701

Schrijver, C. J., Kauristie, K., Aylward, A. D., et al. 2015, AdSpR, 55, 2745 
Schrijver, C. J., Title, A. M., Yeates, A. R., \& DeRosa, M. L. 2013, ApJ, 773, 93

Trichas, M., Gibbs, M., Harrison, R., et al. 2015, Hipparchos, 21, 25

Upton, L., \& Hathaway, D. H. 2014, ApJ, 780, 5

van Ballegooijen, A. A., Priest, E. R., \& Mackay, D. H. 2000, ApJ, 539, 983

Wang, Y.-M., \& Sheeley, N. R. 2002, JGRA, 107, 1302

Wang, Y.-M., \& Sheeley, N. R., Jr. 1990a, ApJ, 365, 372
Wang, Y.-M., \& Sheeley, N. R., Jr. 1990b, ApJ, 355, 726

Wang, Y.-M., \& Sheeley, N. R., Jr. 1992, ApJ, 392, 310

Weinzierl, M., Yeates, A. R., Mackay, D. H., Henney, C. J., \& Arge, C. N. 2016, ApJ, 823, 55

Yang, W. H., Sturrock, P. A., \& Antiochos, S. K. 1986, ApJ, 309, 383

Yeates, A. R., Mackay, D. H., \& van Ballegooijen, A. A. 2008, SoPh, 247, 103

Yeates, A. R., Mackay, D. H., van Ballegooijen, A. A., \& Constable, J. A. 2010, JGRA, 115, 9112 